\title{
THE LEMPERT THEOREM AND THE TETRABLOCK
}

\author{
ARMEN EDIGARIAN, ŁUKASZ KOSIŃSKI, AND WŁODZIMIERZ ZWONEK
}

\begin{abstract}
In the paper we show that the Lempert property (i.e. the equality between the Lempert function and the Carathéodory distance) holds in the tetrablock, a bounded hyperconvex domain which is not biholomorphic to a convex domain. The question whether such an equality holds was posed in 3 .
\end{abstract}

\section{Introduction AND MAin RESUlts}

The paper may be seen as a direct continuation of the study of the geometry of the tetrablock, a domain introduced recently and then studied in a series of papers ([3, 2], [20, [10], [13]).

The tetrablock naturally appears in control engineering and produces problems of a function-theoretic character. We denote the tetrablock by $\mathbb{E}$ and we define it to be the image of the Cartan domain of the first type $\mathcal{R}_{I}:=\mathcal{R}_{I}(2,2)=\{x \in \mathcal{M}(2 \times 2, \mathbb{C}):\|x\|<1\}$, where $\|\cdot\|$ denotes the operator norm of matrices, under the mapping $\pi(x):=\left(x_{11}, x_{22}, \operatorname{det} x\right)$. Note also that $\mathbb{E}$ is the image under $\pi$ of $\mathcal{R}_{I I}:=\mathcal{R}_{I I}(2,2):=\left\{x \in \mathcal{R}_{I}(2,2): x=x^{t}\right\}$ (the Cartan domain of the second type).

In the paper [3] several equivalent definitions of the domain $\mathbb{E}$ are given. Recall two of them

$$
\mathbb{E}=\left\{\left(z_{1}, z_{2}, z_{3}\right) \in \mathbb{C}^{3}:\left|z_{2}-\bar{z}_{1} z_{3}\right|+\left|z_{1} z_{2}-z_{3}\right|+\left|z_{1}\right|^{2}<1\right\}
$$

2000 Mathematics Subject Classification. 32F45.

Key words and phrases. Lempert Theorem, complex geodesic, Lempert function, Carathéodory distance.

The work is partially supported by the grant of the Polish Minister for Science and Higher Education No. N N201 361436.

Part of the paper was prepared while the stay of the Authors at the Erwin Schrödinger International Institute for Mathematical Physics in Vienna during the programme: The dbar-Neumann problem: analysis, geometry and potential theory. 
and

$$
\mathbb{E}=\left\{\left(z_{1}, z_{2}, z_{3}\right) \in \mathbb{C}^{3}:\left|z_{1}-\bar{z}_{2} z_{3}\right|+\left|z_{2}-\bar{z}_{1} z_{3}\right|+\left|z_{3}\right|^{2}<1\right\}
$$

It is proven in [3] that the equality between the Carathéodory distance and the Lempert function of $\mathbb{E}$ with one of the arguments fixed at the origin,

$$
c_{\mathbb{E}}(0, \cdot)=\tilde{k}_{\mathbb{E}}(0, \cdot)
$$

holds on $\mathbb{E}$, which suggests that the equality between both functions holds on $\mathbb{E} \times \mathbb{E}$. The question whether these function are equal was also posed explicitly in [3]. Since both functions are biholomorphically invariant, we also get immediately the equality

$$
c_{\mathbb{E}}(z, \cdot)=\tilde{k}_{\mathbb{E}}(z, \cdot) \text { on } \mathbb{E}
$$

for any $z \in\{\Psi(0): \Psi \in \operatorname{Aut}(\mathbb{E})\}=\{(a, b, a b): a, b \in \mathbb{D}\}$, where Aut $(\mathbb{E})$ is the set of all biholomorphisms of $\mathbb{E}$ (for a description of holomorphic automorphisms of $\mathbb{E}$ see [20] and [13]).

The main purpose of the paper is to show that in fact the above equality holds everywhere in the tetrablock thus solving the problem posed in [3]. In other words we prove

Theorem 1. The equality $c_{\mathbb{E}}=\tilde{k}_{\mathbb{E}}$ holds.

Since the tetrablock is a hyperconvex domain (and thus taut), in order to prove the above theorem it is equivalent to show that for any $\tilde{k}_{\mathbb{E}}$-extremal $f$ there is a function $F \in \mathcal{O}(\mathbb{E}, \mathbb{D})$ such that $F \circ f=\operatorname{id}_{\mathbb{D}}$ where $\mathbb{D}$ denotes the unit disc in $\mathbb{C}$. And this is what we actually do.

Recall that the fundamental Lempert theorem (see [14], 15]) states that the Lempert function and the Carathéodory distance coincide on convex domains (and thus on domains that may be exhausted by domains biholomorphic to convex ones). Nevertheless, very little was known about the converse theorem in a reasonable class of domains (e.g. in the class of bounded and pseudoconvex domains). A few years ago C. Costara, J. Agler and N. J. Young showed that the Lempert theorem would hold in the symmetrized bidisc (see [6], [7], 4]) which is neither biholomorphic to a convex domain (see [7]) nor can be exhausted by domains biholomorphic to convex ones (see [8]). The symmetrized bidisc is a domain in $\mathbb{C}^{2}$ denoted by $\mathbb{G}_{2}$ and given by

$$
\mathbb{G}_{2}:=\left\{(s, p) \in \mathbb{C}^{2}:|s-\bar{s} p|+\left|p^{2}\right|<1\right\} .
$$


For the basic properties of $\mathbb{G}_{2}$ we refer the reader to [4].

Following the ideas in the papers [7] and [8] we show that the tetrablock has the same properties.

Theorem 2. $\mathbb{E}$ cannot be exhausted by domains biholomorphic to convex ones.

The above theorems show that the tetrablock is the second example of that kind. Recall also that the symmetrized bidisc is a $\mathbb{C}$-convex domain (see [17]) Therefore it is natural to pose the question whether $\mathbb{E}$ is biholomorphic to a $\mathbb{C}$-convex domain (or even more, whether it can be exhausted by domains biholomorphic to $\mathbb{C}$-convex domains). And we also may repeat other open questions concerning the $\mathbb{C}$-convexity. Does the Lempert theorem hold for any bounded $\mathbb{C}$-convex domain (see [21])? In fact, since the Lempert Theorem holds for all $C^{2}$-smooth $\mathbb{C}$-convex domains (see [11]), the positive answer to this question would follow from the positive solution of another problem from [21]: Can any bounded $\mathbb{C}$-convex domain be exhausted by $C^{2}$-smooth $\mathbb{C}$-convex domains?

The tetrablock is an example of a bounded $(1,0,1)$-balanced and $(0,1,1)$-balanced pseudoconvex domain; recall that if $m_{1}, \ldots, m_{n}$ are non-negative integers (to avoid trivialities we assume that at least one of $m_{j}$ 's is non-zero) then a domain $D \subset \mathbb{C}^{n}$ is called $\left(m_{1}, \ldots, m_{n}\right)$-balanced if for any $z \in D$ and $\lambda \in \overline{\mathbb{D}}$ the point $\left(\lambda^{m_{1}} z_{1}, \ldots, \lambda^{m_{n}} z_{n}\right)$ lies in $D$. In the proof of Theorem 1 we make use of the following result that has a more general formulation and is interesting for its own.

Theorem 3. Let $D$ be an $\left(m_{1}, \ldots, m_{n}\right)$ balanced pseudoconvex domain. Assume that $\psi$ is a complex geodesic in $D$ and $\psi(\lambda)=\left(\lambda^{m_{1}} \varphi_{1}(\lambda), \ldots, \lambda^{m_{n}} \varphi_{n}(\lambda)\right), \lambda \in \mathbb{D}$, for some $\varphi_{j}$ holomorphic on $\mathbb{D}, j=1, \ldots, n$.

Then $\varphi \in \mathcal{O}(\mathbb{D}, \partial D)$ or $\varphi$ is a complex geodesic in $D$.

Note that one may formulate Theorem 3 replacing the geodesics with $\tilde{k}$-extremals - in this case the proof is immediate.

Acknowledgments The authors express their thanks to the referee for her/his valuable suggestions which essentially improved the quality of the paper.

\section{Definitions, preliminary Considerations And proof of Theorem 3}

First we recall basic definitions of the considered notions. The basic properties in the theory of holomorphically invariant functions may be found in [12]. 
For a domain $D \subset \mathbb{C}^{n}, w, z \in D$, we define the Lempert function

$$
\tilde{k}_{D}(w, z):=\inf \left\{p\left(\lambda_{1}, \lambda_{2}\right): \text { there is } f \in \mathcal{O}(\mathbb{D}, D), f\left(\lambda_{1}\right)=w, f\left(\lambda_{2}\right)=z\right\}
$$

where $p$ denotes the Poincaré distance on $\mathbb{D}$.

If $w \neq z$, then any $f$ as in the definition of $\tilde{k}_{D}$ such that $\tilde{k}_{D}(w, z)=p\left(\lambda_{1}, \lambda_{2}\right)$ is called a $\tilde{k}_{D}$-extremal for $(w, z)$ (or shortly extremal).

We also define the Carathéodory (pseudo)distance by

$$
c_{D}(w, z):=\sup \{p(F(w), F(z)): F \in \mathcal{O}(D, \mathbb{D})\}
$$

It is obvious that $c_{D} \leq \tilde{k}_{D}$. The Lempert Theorem states that if $D$ is convex then $\tilde{k}_{D}=c_{D}$.

The idea of the proof of Theorem 1 is the following. We take any $\tilde{k}_{\mathbb{E}^{-}}$extremal $f$ (and the existence of extremal for any pair of different points from $\mathbb{E}$ follows from the tautness of $\mathbb{E}$ ) and we show that there is a left inverse to $f$, i.e. a mapping $F \in \mathcal{O}(\mathbb{E}, \mathbb{D})$ such that $F \circ f$ is an automorphism (without loss of generality we may require the automorphism to be the identity). In such a case the mapping $f$ is called a complex geodesic. There will be two kinds of possible choices of $F$ depending on the location of the image of $f$. Either the image of $f$ intersects $\mathcal{T}:=\left\{z \in \mathbb{C}^{3}: z_{1} z_{2}=z_{3}\right\}$ or it is disjoint from $\mathcal{T}$. In the second case we can lift the extremal (using the fact that $\left.\pi\right|_{\mathcal{R}_{I I} \backslash \pi^{-1}(\mathcal{T})}$ is a holomorphic covering onto $\mathbb{E} \backslash \mathcal{T}$ ). In the first case we cannot use the lifting coming from the holomorphic covering. Nevertheless, making use of the explicit form of the covering we may lift the extremal to the extremal lying in $\mathcal{R}_{I}$. In both cases all the extremals (which are automatically complex geodesics because of the convexity of $\mathcal{R}_{I}$ and $\mathcal{R}_{I I}$ ) are known. So we have a form of possible extremals. Now the left inverse will be of two possible forms. Either the one considered in several papers in the case the extremal passes through $\mathcal{T}$ (see [3] and [10]) or a function obtained from that in a way described in a more general situation in the proof of Theorem 3. Therefore, we start with the proof of that theorem.

Proof of Theorem [3. It is clear that $\varphi \in \mathcal{O}(\mathbb{D}, \partial D)$ or $\varphi \in \mathcal{O}(\mathbb{D}, D)$. Assume that the second case holds. Let $F \in \mathcal{O}(D, \mathbb{D})$ be such that $F \circ \psi=\operatorname{id}_{\mathbb{D}}$.

We claim that for any $z=\left(z_{1}, \ldots, z_{n}\right) \in D$ there is exactly one $\lambda=\lambda(z) \in \mathbb{D}$ such that $F\left(\lambda^{m_{1}} z_{1}, \ldots, \lambda^{m_{n}} z_{n}\right)=\lambda$. In fact, fix $z \in D$ and consider two functions defined on a neighborhood of $\overline{\mathbb{D}}: \lambda \rightarrow F\left(\lambda^{m_{1}} z_{1}, \ldots, \lambda^{m_{n}} z_{n}\right)$ and $\lambda \rightarrow \lambda$. Since $\left|F\left(\lambda^{m_{1}} z_{1}, \ldots, \lambda^{m_{n}} z_{n}\right)\right|<$ $1=|\lambda|$ for all $\lambda \in \partial \mathbb{D}$, the Rouché theorem implies that the function $\mathbb{D} \ni \lambda \rightarrow \lambda-$ 
$F\left(\lambda^{m_{1}} z_{1}, \ldots, \lambda^{m_{n}} z_{n}\right)$ has exactly one root in $\mathbb{D}$ which finishes the proof of our claim. This allows us to define well a function $G: D \mapsto \mathbb{D}$ with $G(z):=\lambda(z), z \in D$.

Since the graph of $G$ which is equal to

$$
\left\{(z, \lambda) \in D \times \mathbb{D}: F\left(\lambda^{m_{1}} z_{1}, \ldots, \lambda^{m_{n}} z_{n}\right)=\lambda\right\}
$$

is an analytic set (for the notion of analytic sets we refer the reader to [16]) we get that $G$ is holomorphic (see e.g. [16], Chapter V, $\S 1$ ). Moreover, it follows from the definition that $G \circ \varphi(\lambda)=\lambda, \lambda \in \mathbb{D}$, which finishes the proof.

\section{Proof of Theorem $1-$ The CASE $f(\mathbb{D}) \cap \mathcal{T} \neq \varnothing$.}

Let $\Phi_{a}(x)=\left(1-a a^{*}\right)^{-\frac{1}{2}}(x-a)\left(1-a^{*} x\right)^{-1}\left(1-a^{*} a\right)^{\frac{1}{2}}, a, x \in \mathcal{R}_{I}$. It is known (see e.g. [5]) that $\Phi_{a} \in \operatorname{Aut}\left(\mathcal{R}_{I}\right), \Phi_{a}(0)=-a$ and $\Phi_{a}(a)=0$. If additionally $a, x$ are symmetric, then $\Phi_{a}(x)$ is symmetric as well. Therefore, $\Phi_{a} \in \operatorname{Aut}\left(\mathcal{R}_{I I}\right), a \in \mathcal{R}_{I I}$.

It follows from [13] that for any $\psi \in \operatorname{Aut}(\mathbb{E})$ there is a $\Phi \in \operatorname{Aut}\left(\mathcal{R}_{I I}\right)$ such that

$$
\psi \circ \pi(x)=\pi \circ \Phi(x), \quad x \in \mathcal{R}_{I I} .
$$

It is easy to observe that

$$
\Phi=U \Phi_{a} U^{t}
$$

for some $a=\left(\begin{array}{cc}a_{1} & 0 \\ 0 & a_{2}\end{array}\right), a_{1}, a_{2} \in \mathbb{D}$, and $U=\left(\begin{array}{cc}e^{i \theta} & 0 \\ 0 & e^{i \eta}\end{array}\right)$ or $U=\left(\begin{array}{cc}0 & e^{i \theta} \\ e^{i \eta} & 0\end{array}\right)$, $\theta, \eta \in \mathbb{R}$. Direct computations show that the equality (7) remains valid on $\mathcal{R}_{I}$, i.e.

$$
\psi \circ \pi(x)=\pi \circ \Phi(x), \quad x \in \mathcal{R}_{I} .
$$

Note also that it follows from (7) that all automorphisms of $\mathbb{E}$ extend holomorphically onto a neighborhood of $\overline{\mathbb{E}}$.

Put $\tilde{c}=\left(\begin{array}{ll}0 & 0 \\ c & 0\end{array}\right)$, where $c \in \mathbb{D}$. Let us denote

$$
\varphi_{c}(x):=\Phi_{\tilde{c}}(x)=\left(\begin{array}{cc}
\sqrt{1-|c|^{2}} \frac{x_{11}}{1-\bar{c} x_{21}} & \frac{x_{12}+\bar{c} \operatorname{det} x}{1-\bar{c} x_{21}} \\
\frac{x_{21}-c}{1-\bar{c} x_{21}} & \sqrt{1-|c|^{2}} \frac{x_{22}}{1-\bar{c} x_{21}}
\end{array}\right), \quad x=\left(x_{i j}\right) \in \mathcal{R}_{I} .
$$

Note that

$$
\operatorname{det} \varphi_{c}(x)=\frac{\operatorname{det} x+c x_{12}}{1-\bar{c} x_{21}}
$$

We start with the following observation: 
Lemma 4. Let $f: \mathbb{D} \rightarrow \partial \mathbb{E}$ be an analytic disc. If $f(\mathbb{D}) \cap \mathcal{T} \neq \varnothing$, then $f(\mathbb{D}) \subset \mathcal{T}$.

Proof. Using (1) we get

$$
\left|f_{2}-\overline{f_{1}} f_{3}\right|+\left|f_{1} f_{2}-f_{3}\right|=1-\left|f_{1}\right|^{2}
$$

Let $\lambda_{0}$ be such that $f\left(\lambda_{0}\right) \in \mathcal{T}$. Then $\left|f_{2}\left(\lambda_{0}\right)-\overline{f_{1}\left(\lambda_{0}\right)} f_{3}\left(\lambda_{0}\right)\right|=1-\left|f_{1}\left(\lambda_{0}\right)\right|^{2}$. Using the equality $f_{1}\left(\lambda_{0}\right) f_{2}\left(\lambda_{0}\right)=f_{3}\left(\lambda_{0}\right)$ again we infer that

$$
\left|f_{2}\left(\lambda_{0}\right)\right|\left(1-\left|f_{1}\left(\lambda_{0}\right)^{2}\right|\right)=1-\left|f_{1}\left(\lambda_{0}\right)^{2}\right|,
$$

whence $\left|f_{1}\right| \equiv 1$ or $\left|f_{2}\right| \equiv 1$ (recall that $\left|f_{i}\right| \leq 1, i=1,2,3$ ). Assume without loss of generality that $\left|f_{1}\right| \equiv 1$. Making use of (11) we find that $f_{1} f_{2}=f_{3}$.

Definition 5. For a holomorphic mapping $f: \mathbb{D} \rightarrow \mathbb{E}$ put

$$
\nu(f)(\lambda)=\operatorname{ord}_{\lambda}\left(f_{1} f_{2}-f_{3}\right), \quad \lambda \in \mathbb{D} \text {. }
$$

Remark 6. Note that $f(\mathbb{D}) \subset \mathbb{E} \backslash \mathcal{T}$ if and only if $\nu(f) \equiv 0$. Moreover, $\nu$ is invariant under automorphisms of the tetrablock, i.e.

$$
\nu(f) \equiv \nu(\varphi \circ f), \quad \varphi \in \operatorname{Aut}(\mathbb{E}) .
$$

Actually, it follows from (7) that there is an automorphism $\Phi$ of $\mathcal{R}_{I I}$ such that $\varphi(\pi(x))=$ $\pi(\Phi(x))$ for $x \in \mathcal{R}_{I I}$. Moreover, $\Phi$ is of the form (8). Direct calculations show that $\varphi_{1}(x) \varphi_{2}(x)-\varphi_{3}(x)=\left(x_{1} x_{2}-x_{3}\right) e^{2 i(\eta+\theta)}\left(1-\left|a_{1}\right|^{2}\right)\left(1-\left|a_{2}\right|^{2}\right)\left(1-\bar{a}_{1} x_{1}-\bar{a}_{2} x_{2}+\bar{a}_{1} \bar{a}_{2} x_{3}\right)^{-2}, x=$ $\left(x_{1}, x_{2}, x_{3}\right) \in \mathbb{E}$, where $\eta, \theta$ and $a$ are as in (8) . Since $1-\bar{a}_{1} x_{1}-\bar{a}_{2} x_{2}+\bar{a}_{1} \bar{a}_{2} x_{3}=\operatorname{det}\left(1-a^{*} y\right)$, where $y \in \mathcal{R}_{I I}$ is such that $\pi(y)=x$, we see that the function $x \mapsto 1-\bar{a}_{1} x_{1}-\bar{a}_{2} x_{2}+\bar{a}_{1} \bar{a}_{2} x_{3}$ does not vanish on $\mathbb{E}$. This immediately gives (12).

Lemma 7. Let $f: \mathbb{D} \rightarrow \mathbb{E}$ be a holomorphic disc such that $f^{-1}(\mathcal{T}) \neq \varnothing$. Then there is a holomorphic disc $F: \mathbb{D} \rightarrow \overline{\mathcal{R}_{I}}$ such that $f=\pi \circ F$.

Moreover, one of two following possibilities holds:

(a) $F(\mathbb{D}) \subset \mathcal{R}_{I}$

(b) there is an automorphism $\varphi$ of the tetrablock and a holomorphic mapping $\psi: \mathbb{D} \rightarrow \mathbb{D}$ such that $f(\lambda)=\varphi((0,0, \psi(\lambda))), \lambda \in \mathbb{D}$.

Proof. Step 1. First consider the case when $\# f^{-1}(\mathcal{T})=1$. Since the group Aut $(\mathbb{E})$ acts transitively on $\mathcal{T}$, losing no generality we may assume that $f(0)=0$. Then there are 
$n, m \in \mathbb{N}, n+m>0$, such that

$$
f=\left(\lambda^{n} g_{1}, \lambda^{m} g_{2}, \lambda^{n+m} g_{3}\right)
$$

for some holomorphic $g=\left(g_{1}, g_{2}, g_{3}\right): \mathbb{D} \rightarrow \overline{\mathbb{E}}, g(0) \neq 0$. Note that $g(\mathbb{D} \backslash\{0\}) \cap \mathcal{T}=\varnothing$ and $\nu(g)(0)<\nu(f)(0)$.

If $\nu(g)(0)=0$ (i.e. $g(0) \notin \mathcal{T}$ ), then $g_{1} g_{2}-g_{3}$ does not vanish on $\mathbb{D}$. Let $\tilde{g}$ be an analytic square root of $g_{1} g_{2}-g_{3}$. Then the mapping $G=\left(\begin{array}{cc}g_{1} & \tilde{g} \\ \tilde{g} & g_{2}\end{array}\right): \mathbb{D} \rightarrow \overline{\mathcal{R}_{I I}}$ satisfies $g=\pi \circ G$. Put $F(\lambda):=\left(\begin{array}{cc}\lambda^{n} g_{1}(\lambda) & \lambda^{n} \tilde{g}(\lambda) \\ \lambda^{m} \tilde{g}(\lambda) & \lambda^{m} g_{2}(\lambda)\end{array}\right), \lambda \in \mathbb{D}$. Clearly $F: \mathbb{D} \rightarrow \overline{\mathcal{R}_{I}}$ and $f=\pi \circ F$.

If $\nu(g)(0) \neq 0$, then $g(0) \in \mathcal{T}$ and, by Lemma $4, g(\mathbb{D}) \subset \mathbb{E}$. Let $\varphi \in \operatorname{Aut}(\mathbb{E})$ be such that $\varphi(g(0))=0$. There is an analytic disc $h: \mathbb{D} \rightarrow \overline{\mathbb{E}}$ such that $h(0) \neq 0$ and

$$
\varphi \circ g=\left(\lambda^{n_{1}} h_{1}, \lambda^{m_{1}} h_{2}, \lambda^{n_{1}+m_{1}} h_{3}\right)
$$

$n_{1}, m_{1} \in \mathbb{N}, n_{1}+m_{1}>0$. In view of Remark 6

$$
\nu(h)(0)<\nu(\varphi \circ g)(0)=\nu(g)(0)<\nu(f)(0) .
$$

If $\nu(h)(0)=0$ repeating the previous argument we find that there is a mapping $H: \mathbb{D} \rightarrow$ $\overline{\mathcal{R}_{I I}}$ such that $h=\pi \circ H$. Therefore, we may construct a mapping $G_{1}: \mathbb{D} \rightarrow \overline{\mathcal{R}_{I}}$ such that $\varphi \circ g=\pi \circ G_{1}$. Making use of (9) we infer that $g=\pi \circ \widehat{G}$ for some analytic disc $\widehat{G}=\left(\widehat{g}_{i j}\right)$ in $\overline{\mathcal{R}_{I}}$. In particular, $f=\pi \circ F_{1}$, where $F_{1}: \mathbb{D} \rightarrow \overline{\mathcal{R}_{I}}$ is given by the formula $F_{1}(\lambda)=\left(\begin{array}{cc}\lambda^{n_{1}} \widehat{g}_{11}(\lambda) & \lambda^{n_{1}} \widehat{g}_{12}(\lambda) \\ \lambda^{m_{1}} \widehat{g}_{21}(\lambda) & \lambda^{m_{1}} \widehat{g}_{22}(\lambda)\end{array}\right), \lambda \in \mathbb{D}$. If $\nu(h)(0)>0$ we repeat the above procedure (until $\nu=0$ ).

Step 2. In the case when $f^{-1}(\mathcal{T})$ is finite it is sufficient to apply the procedure from the previous step to every point of $f^{-1}(\mathcal{T})$.

Step 3. Now consider the case when that $f^{-1}(\mathcal{T})$ is infinite. If $f(\mathbb{D}) \subset \mathcal{T}$, the statement is clear. In the other case applying Step 2 to the family of analytic $\left.\operatorname{discs} f\right|_{(1-1 / n) \mathbb{D}}, n \in \mathbb{N}$, we find that there are holomorphic mappings $g_{n}:(1-1 / n) \mathbb{D} \rightarrow \overline{\mathcal{R}_{I}}$ such that

$$
f \equiv \pi \circ g_{n} \quad \text { on }(1-1 / n) \mathbb{D} \text {. }
$$

Taking a limit of a subsequence we get a holomorphic $g: \mathbb{D} \rightarrow \overline{\mathcal{R}_{I}}$ such that

$$
f \equiv \pi \circ g
$$

which finishes the proof of the first assertion. 
To prove the second statement assume without loss of generality that $f(0)=0$. Note that $g(0)=\left(\begin{array}{ll}0 & 0 \\ c & 0\end{array}\right)$ or $g(0)=\left(\begin{array}{ll}0 & c \\ 0 & 0\end{array}\right)$ for some $c \in \overline{\mathbb{D}}$. If $c \in \partial \mathbb{D}$ we deduce that $f=(0,0, \psi)$ for some holomorphic mapping $\psi$. In the case when $c$ lies in the unit disc it is clear that $g(0) \in \mathcal{R}_{I}$, whence $g(\mathbb{D}) \subset \mathcal{R}_{I}$.

Recall that any complex geodesic in $\mathcal{R}_{I}$ passing through the origin is of the form

$$
\mathbb{D} \ni \lambda \rightarrow U\left(\begin{array}{cc}
\lambda & 0 \\
0 & Z(\lambda)
\end{array}\right) V \in \mathcal{R}_{I}
$$

where $U, V$ are unitary matrices and $Z: \mathbb{D} \rightarrow \mathbb{D}$ is a holomorphic mapping such that either $Z(\lambda)=\lambda$ or $|Z(\lambda)|<|\lambda|$ for $\lambda \in \mathbb{D} \backslash\{0\}$ (see [1]).

If $f$ is an extremal function in the tetrablock and $g: \mathbb{D} \rightarrow \mathcal{R}_{I}$ is any holomorphic mapping covering $f$ (i.e. $\pi \circ g=f$ ), then $g$ is extremal as well. This simple observation together with Lemma 7 and the description of complex geodesics of the classical Cartan domain of the first type lead to the following statement which is of key importance for our considerations:

Corollary 8. If $f: \mathbb{D} \rightarrow \mathbb{E}$ is an extremal mapping such that $f(0)=0$, then either $f(\mathbb{D}) \subset \mathcal{T}$ or $f(\lambda)=\left(0,0, e^{i \theta} \lambda\right)$ or there are unitary matrices $U, V$ and there is $c \in \mathbb{D}$ such that

$$
f(\lambda)=\pi\left(\varphi_{c}\left(U\left(\begin{array}{cc}
\lambda & 0 \\
0 & Z(\lambda)
\end{array}\right) V\right)\right)
$$

where $\varphi_{c}$ is an automorphism of the Cartan domain of the first type given by the formula (10) and $Z: \mathbb{D} \rightarrow \mathbb{D}$ is a holomorphic mapping. Moreover $|Z(\lambda)|<|\lambda|, \lambda \in \mathbb{D} \backslash\{0\}$, or $Z(\lambda)=\lambda, \lambda \in \mathbb{D}$.

Lemma 9. Let $v=\left(v_{i j}\right) \in \partial \mathcal{R}_{I}$. If $\pi(v) \in \partial \mathbb{E}$, then $\left|v_{12}\right|=\left|v_{21}\right|$.

Proof. Seeking a contradiction suppose that $\left|v_{12}\right| \neq\left|v_{21}\right|$. Put $\tilde{v}=\left(\begin{array}{cc}v_{11} & w \\ w & v_{22}\end{array}\right)$, where $w$ is an arbitrary square root of $v_{12} v_{21}$. Note that it would suffice to show that

$$
\|\tilde{v}\|<\|v\|
$$

Actually, since $\pi(\tilde{v})=\pi(v)$ and $\|v\|=1$, the inequality (15) would imply that $\pi(v) \in \mathbb{E}$. 
Let us denote $\rho:=\|v\|^{2}=\rho\left(v v^{*}\right)$ and $\tilde{\rho}:=\|\tilde{v}\|^{2}=\rho\left(\tilde{v} \tilde{v}^{*}\right)$. Put $d:=\operatorname{det} v=\operatorname{det} \tilde{v}$, $t:=\operatorname{tr}\left(v v^{*}\right)=\left|v_{11}\right|^{2}+\left|v_{12}\right|^{2}+\left|v_{21}\right|^{2}+\left|v_{22}\right|^{2}$ and $\tilde{t}:=\operatorname{tr}\left(\tilde{v} \tilde{v}^{*}\right)=\left|v_{11}\right|^{2}+2\left|v_{12}\right|\left|v_{21}\right|+\left|v_{22}\right|^{2}$. It is clear that $\tilde{t}<t$.

Since $\rho=1 / 2\left(t+\sqrt{t^{2}-4 d}\right)$ and $\tilde{\rho}=1 / 2\left(\tilde{t}+\sqrt{\tilde{t}^{2}-4 d}\right)$, we find that $\tilde{\rho}<\rho$, which proves (15).

Proof of Theorem 1 in the case $f(\mathbb{D}) \cap \mathcal{T} \neq \varnothing$. Let $f$ be an extremal mapping in the tetrablock such that the image of $f$ intersects the set of triangular points. We lose no generality assuming that $f(0)=0$. Let $\tau, \sigma \in \mathbb{D}, \tau \neq \sigma$ be such that $f$ is extremal for $(f(\tau), f(\sigma))$. We aim at showing that $f$ is a complex geodesic.

If $f(\lambda)=\left(0,0, e^{i \theta} \lambda\right), \lambda \in \mathbb{D}$, the statement is clear. The case $f(\mathbb{D}) \subset \mathcal{T}$ follows from [2], Corollary 6.9. Therefore, using Corollary 8, we may assume that $f$ is of the form (14).

First we consider the case when $Z(\lambda)=\lambda, \lambda \in \mathbb{D}$. Then $f(\lambda)=\pi\left(\varphi_{c}(W \lambda)\right), \lambda \in \mathbb{D}$, where $W=U V$ is unitary. Making use of the formula (10) we find that $f=(\alpha, \omega \alpha, \gamma)$, where $\alpha(\lambda)=\sqrt{1-|c|^{2}} w_{11} \lambda /\left(1-\bar{c} \lambda w_{21}\right), \gamma(\lambda)=\left(\operatorname{det} W \lambda^{2}+c \lambda w_{12}\right) /\left(1-\bar{c} \lambda w_{21}\right), \lambda \in \mathbb{D}$, and $\omega \in \partial \mathbb{D}$ is such that $w_{22}=\omega w_{11}$. Since the tetrablock is $(0,1,1)$-balanced we may assume that $\omega=1$.

The descriptions (2) of the tetrablock and (3) of the symmetrized bidisc give us the embedding

$$
\mathbb{G}_{2} \ni(s, p) \mapsto(s / 2, s / 2, p) \in \mathbb{E} .
$$

Since $f$ is extremal, one can see that $\tilde{f}:=(2 \alpha, \gamma)$ is extremal in $\mathbb{G}_{2}$. Therefore, it follows from [4] that $\tilde{f}$ is a geodesic in $\mathbb{G}_{2}$ and its left inverse is given by

$$
F_{a}(s, p)=\frac{2 a p-s}{2-a s}, \quad(s, p) \in \mathbb{G}_{2},
$$

for some $a \in \partial \mathbb{D}$. Put

$$
\Psi_{z}(x):=\frac{z x_{3}-x_{1}}{1-z x_{2}} \quad x \in \mathbb{E}
$$

where $z \in \overline{\mathbb{D}}$, and recall that $\left|\Psi_{z}\right|<1$ on $\mathbb{E}$ whenever $z \in \overline{\mathbb{D}}$ (see [3], Theorem 2.1). It follows from the above considerations that $\Psi_{a}(f(\lambda))=F_{a}(\tilde{f}(\lambda))=\lambda, \lambda \in \mathbb{D}$, so $\Psi_{a}$ is a left inverse of $f$, whence $f$ is a complex geodesic.

Now we focus on the case when $|Z(\lambda)|<|\lambda|$ for $\lambda \in \mathbb{D} \backslash\{0\}$ and $c \neq 0$. It is seen that there is an open neighborhood $D$ of $\overline{\mathbb{D}}$ and a holomorphic, non-rational mapping $W: D \rightarrow \mathbb{C}$ such that $W(\mathbb{D}) \subset \mathbb{D}, W(\tau)=Z(\tau)$ and $W(\sigma)=Z(\sigma)$ (note that we do not demand $W(0)=0)$. 
Put $g(\lambda)=\pi\left(\varphi_{c}\left(U\left(\begin{array}{cc}\lambda & 0 \\ 0 & W(\lambda)\end{array}\right) V\right)\right), \lambda \in \mathbb{D}$. Then $g$ is also an extremal function in the tetrablock (as $g(\sigma)=f(\sigma), g(\tau)=f(\tau)$ ). Therefore $g$ is almost proper, that is $g^{*}(\lambda) \in \partial \mathbb{E}\left(g^{*}\right.$ denotes a nontangential limit of the mapping $g$ ) for almost all $\lambda \in \partial \mathbb{D}$ w.r.t the Lebesgue measure on the unit circle (see e.g. [9]). Since $g$ is holomorphic in a neighborhood of $\overline{\mathbb{D}}$, the almost properness means that $g(\partial \mathbb{D}) \subset \partial \mathbb{E}$.

It follows from Lemma 9 that

$$
\left|\lambda u_{21} v_{11}+W(\lambda) u_{22} v_{21}-c\right|=\left|\lambda u_{11} v_{12}+W(\lambda) u_{12} v_{22}+\bar{c} \lambda W(\lambda) \operatorname{det} U \operatorname{det} V\right|
$$

$\lambda \in \partial \mathbb{D}$. We claim that there are finite Blaschke products $B_{1}, B_{2}$ such that $\left|B_{1}(0)\right|+$ $\left|B_{2}(0)\right| \neq 0$ and

$$
B_{1}(\lambda)\left(\lambda u_{21} v_{11}+W(\lambda) u_{22} v_{21}-c\right)=B_{2}(\lambda)\left(\lambda u_{11} v_{12}+W(\lambda) u_{12} v_{22}+\bar{c} \lambda W(\lambda) e^{i \theta}\right)
$$

$\lambda \in \mathbb{D}$, where $e^{i \theta}=\operatorname{det} U \operatorname{det} V$.

To prove the existence of such Blaschke products take any $f_{1}, f_{2} \in \mathcal{O}(D)$ not vanishing identically and satisfying $\left|f_{1}\right|=\left|f_{2}\right|$ on $\partial \mathbb{D}$. Put $H(\lambda):=\left(\lambda-\lambda_{1}\right) \cdots\left(\lambda-\lambda_{N}\right), \lambda \in D$, where $\lambda_{1}, \ldots, \lambda_{N}$ are common roots of $f_{1}$ and $f_{2}$ lying in $\overline{\mathbb{D}}$ and counted with multiplicities. Since $\left|f_{1}\right|=\left|f_{2}\right|$ on $\partial \mathbb{D}$ we see that $f_{1} / H$ and $f_{2} / H$ do not vanish on $\partial \mathbb{D}$. Therefore, there are finite Blaschke products $\tilde{B}_{i}, i=1,2$, with no common zeros such that that $F_{i}:=f_{i} /\left(H \tilde{B}_{i}\right)$ is holomorphic on a neighborhood of $\overline{\mathbb{D}}$ and does not vanish there, $i=1,2$. Since $\left|\tilde{B}_{i}\right|=1$ on $\partial \mathbb{D}$ we get that $\left|F_{1}\right|=\left|F_{2}\right|$ on $\partial \mathbb{D}$. From this we immediately get that $F_{2} / F_{1}$ is constant - apply the maximum principle to $F_{1} / F_{2}$ and $F_{2} / F_{1}$. Let $F_{2}=\omega F_{1}$, where $|\omega|=1$. Then $f_{1}=F_{1} H \tilde{B}_{1}, F_{2}=\omega F_{1} H \tilde{B}_{2}$, and $\tilde{B}_{1}, \tilde{B}_{2}$ have no common zeros. Putting $B_{1}:=\omega \tilde{B}_{2}$ and $B_{2}:=\tilde{B}_{1}$ we obtain Blaschke products satisfying the desired claim.

Since $W$ is non-rational we infer that

$$
\begin{aligned}
B_{1}(\lambda) \lambda u_{21} v_{11}-c B_{1}(\lambda) & =B_{2}(\lambda) \lambda u_{11} v_{12} \\
B_{1}(\lambda) u_{22} v_{21} & =B_{2}(\lambda) u_{12} v_{22}+B_{2}(\lambda) \bar{c} \lambda e^{i \theta}, \lambda \in \overline{\mathbb{D}} .
\end{aligned}
$$

Putting $\lambda=0$ we get $B_{1}(0)=0$. Since $B_{2}(0) \neq 0$ we get that $u_{12} v_{22}=0$.

If $u_{12}=0$, then $u_{21}=0$ and $\left|u_{11}\right|=\left|u_{22}\right|=1$. Putting it to (17) and taking $|\lambda|=1$ we find that $\left|v_{12}\right|=|c|$. Easy computations give: $\left|v_{11}\right|=\left|v_{22}\right|=\sqrt{1-|c|^{2}}$ and $\left|v_{21}\right|=|c|$. We want to show that $f$ is a complex geodesic. Note that making use of the fact that the tetrablock is $(1,0,1)$ - and $(0,1,1)$-balanced it suffices to get the statement under the 
additional assumption that $u_{11}=u_{22}=1$. Using similar argument we see that it is enough to consider that case $v_{11}=\sqrt{1-|c|^{2}}$ and $v_{12}=|c|$. Then

$$
V=\left(\begin{array}{cc}
\sqrt{1-|c|^{2}} & |c| \\
-\omega|c| & \omega \sqrt{1-|c|^{2}}
\end{array}\right)
$$

for some $\omega$ from the unit circle. Replacing $Z$ with $\omega Z$ we may clearly assume that $\omega=1$. Now, after some simple calculations one can deduce that

$$
f(\lambda)=\left(\frac{\left(1-|c|^{2}\right) \lambda}{1+\bar{c}|c| Z(\lambda)}, \frac{\left(1-|c|^{2}\right) Z(\lambda)}{1+\bar{c}|c| Z(\lambda)}, \lambda \frac{Z(\lambda)+c|c|}{1+\bar{c}|c| Z(\lambda)}\right), \quad \lambda \in \mathbb{D} .
$$

Therefore $f$ is a complex geodesic (it just of the form (2) in Theorem 2 in [10], with permuted two first variables, $\omega_{2}=1, \omega_{1} \in \partial \mathbb{D}$ such that $c=-\omega_{1}|c|, C=|c|^{2}$ and $\left.\varphi(\lambda)=\left(\bar{\omega}_{1} Z(\lambda)-|c|^{2}\right) /\left(1-|c|^{2} \bar{\omega}_{1} Z(\lambda)\right)\right)$. If $v_{22}=0$ we proceed similarly.

Let us focus on the case $c=0$ and $|Z(\lambda)|<|\lambda|, \lambda \in \mathbb{D} \backslash\{0\}$. First note that we may assume that $Z$ extends holomorphically to a neighborhood of $\mathbb{D}$. Let $h$ be a holomorphic function in a heighborhood of $\mathbb{D}$ such that $Z(\lambda)=\lambda h(\lambda), h(\mathbb{D}) \subset \mathbb{D}$. Replacing $Z$ with a non-rational $W: \mathbb{D} \rightarrow \mathbb{D}$ (holomorphic on a neighborhood of $\overline{\mathbb{D}}$ ) such that $W(\sigma)=Z(\sigma)$ and $W(\tau)=Z(\tau)$, making use of Lemma 9 and repeating the argument with Blaschke products (17) we find that $\left|u_{21} v_{11}\right|=\left|u_{11} v_{12}\right|$ and $\left|u_{22} v_{21}\right|=\left|u_{12} v_{22}\right|$. Since $U$ and $V$ are unitary we deduce from these equalities that

$$
\left|u_{i j}\right|=\left|v_{i j}\right|, \quad 1 \leq i, j \leq 2 .
$$

Put

$$
\Phi(\lambda):=U\left(\begin{array}{cc}
\lambda & 0 \\
0 & Z(\lambda)
\end{array}\right) V=\left(\begin{array}{cc}
\lambda u_{11} v_{11}+Z(\lambda) u_{12} v_{21} & \lambda u_{11} v_{12}+Z(\lambda) u_{12} v_{22} \\
\lambda u_{21} v_{11}+Z(\lambda) u_{22} v_{21} & \lambda u_{21} v_{12}+Z(\lambda) u_{22} v_{22}
\end{array}\right)
$$

for $\lambda$ lying in some neighborhood of $\overline{\mathbb{D}}$. Obviously $\Phi(\mathbb{D}) \subset \mathcal{R}_{I}$. Define

$$
\Psi(\lambda)=\left(\begin{array}{cc}
\lambda u_{11} v_{11}+Z(\lambda) u_{12} v_{21} & \lambda^{2} u_{11} v_{12}+\lambda Z(\lambda) u_{12} v_{22} \\
u_{21} v_{11}+h(\lambda) u_{22} v_{21} & \lambda u_{21} v_{12}+Z(\lambda) u_{22} v_{22}
\end{array}\right)
$$

for $\lambda$ from some neighborhood of $\overline{\mathbb{D}}$. Note that $\|\Psi(\lambda)\|=1$ for $\lambda \in \partial \mathbb{D}$ (as $\Psi \Psi^{*}$ and $\Phi \Phi^{*}$ have the same eigenvalues on $\partial \mathbb{D})$ and $\Psi(0) \in \mathcal{R}_{I}$. A standard argument implies that $\Psi$ maps the unit disc into $\mathcal{R}_{I}$ (apply the maximum principle to the subharmonic function $\log \|\Psi(\cdot)\|)$. Observe that $f=\pi \circ \Psi$, whence $\Psi$ is a complex geodesic in $\mathcal{R}_{I}$, as well. Denote $c^{\prime}=-u_{21} v_{11}-h(0) u_{22} v_{21}$. If $c^{\prime}=0$ then $u_{21} v_{11}=0$ (remember that $|h(0)|<1$ and use the equality $\left|u_{21} v_{11}\right|=\left|u_{22} v_{21}\right|$ ), whence $U$ and $V$ are diagonal or anti-diagonal. Then, 
it is easy to observe that $f$ is a complex geodesic (more precisely, up to a permutation of two fist components the mapping $f$ is of the form $f(\lambda)=\left(\omega_{1} \lambda, \omega_{2} Z(\lambda), \omega_{1} \omega_{2} Z(\lambda)\right), \lambda \in \mathbb{D}$, for some $\left.\omega_{1}, \omega_{2} \in \partial \mathbb{D}\right)$.

If $c^{\prime} \neq 0$, then $u_{21} v_{11} \neq 0$. Moving $\Psi(0)$ to the origin and making use of the description of complex geodesics in $\mathcal{R}_{I}$ we infer that there are unitary matrices $U_{1}, V_{1}$ and a mapping $Z_{1}$ defined on $\mathbb{D}$, such that

$$
\Psi(\lambda)=\varphi_{c^{\prime}}\left(U_{1}\left(\begin{array}{cc}
\lambda & 0 \\
0 & Z_{1}(\lambda)
\end{array}\right) V_{1}\right), \quad \lambda \in \mathbb{D}
$$

and $\left|Z_{1}(\lambda)\right|<|\lambda|$ for $\lambda \in \mathbb{D} \backslash\{0\}$ or $Z_{1}(\lambda)=\lambda, \lambda \in \mathbb{D}$. Now we are in a position that allows us to apply the cases already solved (either $Z(\lambda)=\lambda$ for $\lambda \in \mathbb{D}$ or $c \neq 0$ and $|Z(\lambda)|<|\lambda|$ for $\lambda \in \mathbb{D} \backslash\{0\})$.

\section{Proof of Theorem $1-$ The CASe $f(\mathbb{D}) \cap \mathcal{T}=\varnothing$.}

Let $f: \mathbb{D} \rightarrow \mathbb{E}$ be an extremal such that $f(\mathbb{D}) \cap \mathcal{T}=\varnothing$. Then there exists a geodesic $\tilde{f}: \mathbb{D} \rightarrow \mathcal{R}_{I I}$ such that $f=\pi \circ \tilde{f}$. Making use of the form of automorphisms of $\mathbb{E}$ without loss of generality we may assume that $f$ is a $\tilde{k}_{\mathbb{E}^{-}}$extremal for $(f(0), f(\sigma))$ and $f(0)=\left(0,0,-\beta^{2}\right)$.

Any complex geodesic in $\mathcal{R}_{I I}$ passing through the origin can be written as

$$
\varphi(\lambda)=U\left(\begin{array}{cc}
\lambda & 0 \\
0 & Z(\lambda)
\end{array}\right) U^{t}
$$

where $U$ is a unitary matrix and $Z: \mathbb{D} \rightarrow \mathbb{D}$ is a holomorphic mapping such that $Z(0)=0$. Moreover, $|Z(\lambda)|<|\lambda|, \lambda \in \mathbb{D} \backslash\{0\}$, or $Z(\lambda)=\lambda$ (see [1]). Assume that

$$
U=\left(\begin{array}{ll}
a & b \\
c & d
\end{array}\right)
$$

where $|a|^{2}+|b|^{2}=|c|^{2}+|d|^{2}=1$ and $a \bar{c}+b \bar{d}=0$. After some simple calculations we get

$$
\varphi(\lambda)=\left(\begin{array}{cc}
a^{2} \lambda+b^{2} Z(\lambda) & a c \lambda+b d Z(\lambda) \\
a c \lambda+b d Z(\lambda) & c^{2} \lambda+d^{2} Z(\lambda)
\end{array}\right)
$$

Put $A(\lambda)=a^{2} \lambda+b^{2} Z(\lambda), B(\lambda)=a c \lambda+b d Z(\lambda)$, and $C(\lambda)=c^{2}+d^{2} Z(\lambda)$. We "move" now this geodesic to $\left(\begin{array}{cc}0 & \beta \\ \beta & 0\end{array}\right)$ and get the following 
Proposition 10. Let $f: \mathbb{D} \rightarrow \mathbb{E}$ be an extremal mapping for $(f(0), f(\sigma))$ such that $f(0)=$ $\left(0,0,-\beta^{2}\right)$ and $f(\mathbb{D}) \cap \mathcal{T}=\varnothing$. Then there exist $a, b, c, d \in \overline{\mathbb{D}}$ with $|a|^{2}+|b|^{2}=|c|^{2}+|d|^{2}=1$ and $a \bar{c}+b \bar{d}=0$ such that

$$
f(\lambda)=\left(\frac{A(\lambda)\left(1-\beta^{2}\right)}{\Delta(\lambda)}, \frac{C(\lambda)\left(1-\beta^{2}\right)}{\Delta(\lambda)}, \frac{A(\lambda) C(\lambda)-(B(\lambda)+\beta)^{2}}{\Delta(\lambda)}\right),
$$

where $A, B, C$ are defined as above and $\Delta(\lambda)=(1+\beta B(\lambda))^{2}-A(\lambda) C(\lambda) \beta^{2}$.

We show that under the above assumptions the extremal $f$ has its left inverse. First note that the following equations are satisfied: $|a|=|d|,|b|=|c|$ and $|a d-b c|=1$. Note also that we may always assume that $Z(\lambda)=\mu \lambda$ for some $|\mu| \leq 1$. Actually, if the considered mapping is extremal with some $Z$ as above then it will also be extremal with $Z(\lambda)=\mu \lambda$ where $\mu=Z^{\prime}(0)$. If the new considered extremal intersects $\mathcal{T}$ then in view of the previous considerations we already know that it is a complex geodesic. Therefore, we lose no generality assuming that the extremal omitting $\mathcal{T}$ is the one with $Z(\lambda)=\mu \lambda$.

We want to get some relations on the numbers $a, b, c, d$ and $Z$ (equivalently, $\mu$ ) that allow us to describe the mappings as in Proposition 10.

When does the equality $f_{1} f_{2}=f_{3}$ hold at some point of $\mathbb{D}$ (in other words we want to see when $f(\mathbb{D}) \cap \mathcal{T}=\varnothing)$ ?

$$
\frac{A C\left(1-\beta^{2}\right)^{2}}{\triangle^{2}}=\frac{A C-(B+\beta)^{2}}{\triangle}
$$

which is equivalent to

$$
A C \beta=(B+\beta)(1+\beta B) .
$$

Consequently,

$$
\beta \lambda Z(a d-b c)^{2}-\left(1+\beta^{2}\right)(\lambda a c+Z b d)-\beta=0 .
$$

Recall that the Cohn criterion (see e.g. [18] ) gives that the equation $a_{0} \lambda^{2}+a_{1} \lambda+a_{2}=0$ has both solutions in $\mathbb{C} \backslash \mathbb{D}$ iff $\left|a_{2}\right| \geq\left|a_{0}\right|$ and $\left|\bar{a}_{0} a_{1}-a_{2} \bar{a}_{1}\right| \leq\left.|| a_{0}\right|^{2}-\left|a_{2}\right|^{2} \mid$.

When we apply it to our situation $(Z(\lambda)=\mu \lambda)$ we get that $f$ is as desired iff

$$
\left(1+\beta^{2}\right)\left|\bar{\mu}(\bar{a} \bar{d}-\bar{b} \bar{c})^{2}(a c+\mu b d)+(\bar{a} \bar{c}+\bar{\mu} \bar{b} \bar{d})\right| \leq \beta\left(1-|\mu|^{2}\right) .
$$


Then elementary calculations give that the last inequality (remember about the existing relations) is equivalent to $|c||d|\left(1+\beta^{2}\right) \leq \beta$.

It is sufficient to show that we have the left inverse under the sharp inequality.

In view of Theorem 3 it is sufficient to show that for some $\gamma \in \mathbb{D}$ and $|\tau|=1$ the function

$$
g: \mathbb{D} \ni \lambda \mapsto\left(\tau \frac{\lambda-\gamma}{1-\bar{\gamma} \lambda} f_{1}(\lambda), f_{2}(\lambda), \tau \frac{\lambda-\gamma}{1-\bar{\gamma} \lambda} f_{3}(\lambda)\right) \in \mathbb{E}
$$

is a geodesic.

Let $F(z):=\frac{z_{3}-z_{2}}{z_{1}-1}, z \in \mathbb{E}$. We shall prove that by the proper choice of $\tau$ and $\gamma$ the function $h:=F \circ g$ is an automorphism of $\mathbb{D}$. But it is sufficient, by the Schwarz-Pick Lemma to show that $\left|h^{\prime}(0)\right|=1-|h(0)|^{2}$.

But $h(0)=-\tau \gamma \beta^{2}$ and

$$
\begin{aligned}
& h^{\prime}(0)=\tau \beta^{2}\left(1-|\gamma|^{2}\right)-2 \tau \beta \gamma(a c+b d \mu)\left(1-\beta^{2}\right)+ \\
& \quad\left(c^{2}+d^{2} \mu\right)\left(1-\beta^{2}\right)+\left(c^{2}+d^{2} \mu\right)\left(1-\beta^{2}\right)+\tau^{2} \gamma^{2}\left(a^{2}+b^{2} \mu\right)\left(1-\beta^{2}\right) \beta^{2} .
\end{aligned}
$$

Consequently,

$$
h^{\prime}(0)=\left(1-\beta^{2}\right)\left((c-\tau \beta \gamma a)^{2}+\mu(d-b \tau \beta \gamma)^{2}\right)+\tau \beta^{2}\left(1-|\gamma|^{2}\right) .
$$

We choose $|\tau|=1$ and $\gamma \in \mathbb{D}$ such that $d=b \tau \beta \gamma$ and $\left|h^{\prime}(0)\right|=\left|\left(1-\beta^{2}\right)\right| c-\left.\tau \beta \gamma a\right|^{2}+$ $\beta^{2}\left(1-|\gamma|^{2}\right)$, which is possible under the assumption $|d|^{2}<|b|^{2} \beta^{2}$, which is equivalent $\left(|b|=|c|\right.$ and $\left.|c|^{2}+|d|^{2}=1\right)$ to $\frac{1}{1+\beta^{2}}<|c|^{2}$. And the last inequality is equivalent to $|c|^{2}\left(1-|c|^{2}\right)<\frac{\beta^{2}}{\left(1+\beta^{2}\right)^{2}}$.

\section{5. $\mathbb{E}$ CANNOT BE EXHAUSTED BY DOMAINS BIHOLOMORPHIC TO CONVEX ONES}

In this Section we prove Theorem 2 .

For $z \in \mathbb{C}^{3}$ put $\rho(z):=\max \left\|\left(\left.\pi\right|_{\mathcal{R}_{I I}}\right)^{-1}(z)\right\|$. The properness of $\left.\pi\right|_{\mathcal{R}_{I I}}$ implies that $\rho$ is plurisubharmonic.

Proof of Theorem 2. For any $\epsilon \in(0,1)$ we define $G_{\epsilon}:=\left\{z \in \mathbb{C}^{3}: \rho(z)<1-\epsilon\right\}$. Assume that $U_{\epsilon}$ is a neighborhood of $\overline{G_{\epsilon}}$ and $f_{\epsilon}: U_{\epsilon} \mapsto V_{\epsilon}$ where $V_{\epsilon}$ is a convex domain. Without loss of generality we may assume that $0 \in V_{\epsilon}, V_{\epsilon}$ is a convex domain, $f_{\epsilon}(0)=0, f_{\epsilon}^{\prime}(0)=\mathrm{id}$. Denote $\varphi_{\lambda}(z):=\left(\lambda z_{1}, \lambda z_{2}, \lambda^{2} z_{3}\right), \lambda \in \mathbb{C}, z \in \mathbb{E}$.

Fix $w=\left(w_{1}, w_{2}, w_{3}\right), z=\left(z_{1}, z_{2}, z_{3}\right) \in \mathbb{C}^{3}$ and $r \in[0,1]$. Put 
(1) $R:=\max \{\rho(w), \rho(z)\}$,

(2) $g_{\epsilon}(\lambda):=f_{\epsilon}^{-1}\left(r f_{\epsilon}\left(\varphi_{\lambda}(w)\right)+(1-r) f_{\epsilon}\left(\varphi_{\lambda}(z)\right)\right)$.

Note that $g_{\epsilon}(0)=0$ and that $g_{\epsilon}$ is well-defined for $|\lambda|<(1-\epsilon) / R$. Moreover, $\rho\left(g_{\epsilon}(\lambda)\right) \leq 1$ for any $|\lambda|<(1-\epsilon) / R$. Put $h_{\epsilon}(\lambda):=\varphi_{1 / \lambda}\left(g_{\epsilon}(\lambda)\right)$. Then $h_{\epsilon}: \mathbb{D}(0,(1-\epsilon) / R) \backslash\{0\} \mapsto \mathbb{C}^{3}$ is a holomorphic mapping. Then simple calculations show the following properties

(1) $\left(g_{\epsilon}\right)_{j}^{\prime}(0)=r w_{j}+(1-r) z_{j}, j=1,2$,

(2) $\left(g_{\epsilon}\right)_{3}^{\prime}(0)=0$.

Consequently, $h_{\epsilon}$ extends holomorphically to 0 . More calculations show that

$$
\begin{aligned}
\left(g_{\epsilon}\right)_{3}^{\prime \prime}(0)= & 2\left(r w_{3}+(1-r) z_{3}\right)+\frac{\partial^{2}\left(f_{\epsilon}\right)_{3}}{\partial z_{1}^{2}}(0) r(1-r)\left(w_{1}-z_{1}\right)^{2}+ \\
& \frac{\partial^{2}\left(f_{\epsilon}\right)_{3}}{\partial z_{2}^{2}}(0) r(1-r)\left(w_{2}-z_{2}\right)^{2}+2 \frac{\partial^{2}\left(f_{\epsilon}\right)_{3}}{\partial z_{1} \partial z_{2}}(0) r(1-r)\left(w_{1}-z_{1}\right)\left(w_{2}-z_{2}\right) .
\end{aligned}
$$

Define

$$
s_{\epsilon}:=\frac{1}{2} \frac{\partial^{2}\left(f_{\epsilon}\right)_{3}}{\partial z_{1}^{2}}(0), t_{\epsilon}:=\frac{1}{2} \frac{\partial^{2}\left(f_{\epsilon}\right)_{3}}{\partial z_{2}^{2}}(0), u_{\epsilon}:=\frac{\partial^{2}\left(f_{\epsilon}\right)_{3}}{\partial z_{1} \partial z_{2}}(0)
$$

Then

$$
\begin{array}{r}
h_{\epsilon}(0)=\left(r w_{1}+(1-r) z_{1}, r w_{2}+(1-r) z_{2}, r w_{3}+(1-r) z_{3}+s_{\epsilon} r(1-r)\left(w_{1}-z_{1}\right)^{2}+\right. \\
\left.t_{\epsilon} r(1-r)\left(w_{2}-z_{2}\right)^{2}+u_{\epsilon} r(1-r)\left(w_{1}-z_{1}\right)\left(w_{2}-z_{2}\right)\right) .
\end{array}
$$

By the maximum principle

$$
\rho\left(h_{\epsilon}(\lambda)\right)=\rho\left(\varphi_{1 / \lambda}\left(g_{\epsilon}(\lambda)\right)=\frac{1}{|\lambda|} \rho\left(g_{\epsilon}(\lambda)\right) \leq \frac{1}{|\lambda|} .\right.
$$

Hence, $\rho\left(h_{\epsilon}(0)\right) \leq \frac{R}{1-\epsilon}$.

Our next aim is to show that

$$
\lim _{\epsilon \rightarrow 0} s_{\epsilon}=\lim _{\epsilon \rightarrow 0} t_{\epsilon}=\lim _{\epsilon \rightarrow 0} u_{\epsilon}=0 .
$$

Note that the equalities (36) imply that $\rho(r w+(1-r) z) \leq \max \{\rho(w), \rho(z)\}$ for all $w, z \in \mathbb{C}^{3}$, which contradicts the non-convexity of $\mathbb{E}$.

We are just left with the proof of the above equalities. 
Put $r=\frac{1}{2}$. For the proof of the convergence of $s_{\epsilon}$ consider two points $w=(1,1,1)$, $z=(-1,1,-1)$. Putting them to (34) and (35) we find that $\rho\left(0,1, s_{\epsilon}\right) \leq 1 /(1-\epsilon)$. This inequality implies that $s_{\epsilon} \rightarrow 0$.

Similarly, putting $w=(1,1,1)$ and $z=(1,-1,-1)$ one can show that $t_{\epsilon} \rightarrow 0$.

Finally, taking $z=(\zeta, \zeta, \zeta)$ and $w=(-\zeta,-\zeta, \zeta)$, where $|\zeta|=1$ is such that $u_{\epsilon} \zeta=\left|u_{\epsilon}\right|$ we find that $\rho\left(0,0, \zeta\left(1+\zeta t_{\epsilon}+\zeta s_{\epsilon}+\left|u_{\epsilon}\right|\right)\right)<1 /(1-\epsilon)$. Making use of just proven two equalities we get the equality $\lim _{\epsilon \rightarrow 0} u_{\epsilon}=0$.

\section{REFERENCES}

[1] M. AвAтE, The complex geodesics of non-hermitian symmetric spaces, Universiti degli Studi di Bologna, Dipartamento di Matematica, Seminari di geometria, 1991-1993, 1-18.

[2] A. A. Abouhajar, Function theory related to $H^{\infty}$ control, Ph.D. thesis, Newcastle University, 2007.

[3] A. A. Abouhajar, M. C. White \& N. J. Young, A Schwarz lemma for a domain related to mu-synthesis, Journal of Geometric Analysis, 17(4), 2007, 717-750.

[4] J. Agler \& N. J. Young, The hyperbolic geometry of the symmetrized bidisc, J. Geom. Anal. 14 (2004), no. 3, 375-403.

[5] G. Bassanelli, On holomorphic automorphisms of the unit ball of rectangular, complex matrices. Ann. Mat. Pura Appl. (4) 133 (1983), 159-175.

[6] C. Costara, Le probleme de Nevanlinna-Pick spectral, Ph.D. thesis, Université Laval, 2004.

[7] C. Costara, The symmetrized bidisc and Lempert's theorem, Bull. London Math. Soc. 36 (2004), no. $5,656-662$.

[8] A. Edigarian, A note on C. Costara's paper: "The symmetrized bidisc and Lempert's theorem" [Bull. London Math. Soc. 36 (2004), no. 5, 656-662], Ann. Polon. Math. 83 (2004), no. 2, 189-191.

[9] A. Edigarian \& P. Kuiś, Almost Properness of Extremal Mappings, Bull. Polish Acad. Sci. Math., 57 (2) 2009, 129-133.

[10] A. Edigarian \& W. Zwonek, Schwarz lemma for the tetrablock, Bull. Lond. Math. Soc. 41 (2009), no. $3,506-514$.

[11] D. Jacquet, $\mathbb{C}$-convex domains with $C^{2}$ boundary, Complex Var. Elliptic Equ. 51 (2006), no. 4, 303-312.

[12] M. Jarnicki \& P. Pflug, Invariant Distances and Metrics in Complex Analysis, De Gruyter Expositions in Mathematics 9, 1993.

[13] Ł. Kosiński, Geometry of quasi-circular domains and applications to tetrablock, Proc. Amer. Math. Soc. 139 (2011), 559-569.

[14] L. Lempert, La métrique de Kobayashi et la représentation des domaines sur la boule, Bull. Soc. Math. France 109 (1981), no. 4, 427-474.

[15] L. LEMPERT, Holomorphic retracts and intrinsic metrics in convex domains, Anal. Math. 8 (1982), no. 4, 257-261. 
[16] S. Łojasiewicz, Introduction to Complex Analytic Geometry, Birkhäuser Verlag, 1991.

[17] N. Nikolov, P. Pflug \& W. Zwonek, An example of a bounded $\mathbb{C}$-convex domain which is not biholomorphic to a convex domain, Math. Scand. 102 (2008), no. 1, 149-155.

[18] Q. I. Rahman \& G. Schmeisser, Analytic Theory of Polynomials, London Math. Soc. Monogr. (N.S.) 26, Oxford Univ. Press, Oxford, 2002.

[19] W. Rudin, Real and Complex Analysis, 2nd ed., McGraw-Hill, New York, 1974.

[20] N. J. Young, The automorphism group of the tetrablock, Journal of the London Mathematical Society $77(3)$ (2008), 757-770.

[21] S. V. Znamenskit, Seven problems on $\mathbb{C}$-convexity, Complex analysis in modern mathematics (Russian), 123-131, FAZIS, Moscow, 2001.

Institute of Mathematics, Faculty of Mathematics and Computer Science, Jagiellonian University, Łojasiewicza 6, 30-348 Kraków, Poland

E-mail address: Armen.Edigarian@im.uj.edu.pl

E-mail address: Lukasz.Kosinski@im.uj.edu.pl

E-mail address: Wlodzimierz.Zwonek@im.uj.edu.pl 